\title{
APPROACH OF PYRROLO[4,3,2-de] QUINOLINE ALKALOID STRUCTURE
}

\author{
N. Roue 19 and R. Barret ${ }^{2 *}$
}

1- UPRESA 6013-Laboratoire de Chimie Therapeutique, Faculte de Pharmacie, 51, rue CognacqJay. 51100 Reims-France.

2-Laboratoire de Chimie Therapeutique, Faculte de Pharmacie, 8, Avenue Rockefeller, 69373 Lyon Cedex 08 - France - e-mail : roland.barret@rockefeller-univ.lyon1.fr

\begin{abstract}
The oxidation of some indolines into indoles with palladium in the presence of ammonium formate is studied with the aim to obtain precursors of indoloquinoline alkaloids.
\end{abstract}

The tetrahydropyrrolo-[4,3,2-de]quinoline 1 structure is found in several alkaloids such as damirone A and B 2, isolated from sponges ${ }^{1}$, and haematopodin 3 , isolated from fungi?<smiles>c1cc2c3c(c[nH]c3c1)CCN2</smiles>

1<smiles>[R]n1cc2c3c1C(=O)C(=O)C=C3N(C)CC2</smiles>

2<smiles>O=C1C=C2c3c(c[nH]c3C1=O)CC1OCCCN21</smiles>

3

$$
\begin{aligned}
& \mathrm{R}=\mathrm{CH}_{3} \text { : damirone } \mathrm{A} \quad \text { haematopodin } \\
& \mathrm{R}=\mathrm{H} \text { : damirone } \mathrm{B}
\end{aligned}
$$

Scheme 1

Recently we have described the synthesis of 1,3,4,5-tetrahydropyrrolo-[4,3,2-de]quinoline $1^{3}$ from 4-nitroindole. Thus, in this Short Communication, we used this strategy to obtain methoxy synthetic precursors of 2 and 3 , and we described our first tries to obtain the oxazine cycle of 3 .

4-Nitroindoles 4a-c were synthesized by Bergman procedure ${ }^{4}$. 4-Nitro-7-methoxyindoles 4d-f were synthesized by nitration of methoxyindoles as we have previously described 5 . 5a-f were obtained by condensation of oxalyl chloride and then treatment with methanol or by modified Vilsmeier reaction ${ }^{3,6}$; 5 a-f were reduced into indolines $6 \mathbf{6}-\mathbf{f}$ by $\mathrm{HSiEt}_{3}$ (3 equivalents) in trifluroacetic acid. Lactams 7 a- $\mathrm{f}$ were obtained by reduction of nitro function (Pd-C 10\%- $\mathrm{H}_{2}, 5$ bars or $\mathrm{PtO}_{2}-\mathrm{H}_{2}$, atmospheric pressure or $\mathrm{SnCl}_{2}-12 \mathrm{~N} \mathrm{HCl}$, reflux temperature, 3 hours) and heating at reflux temperature in toluene (Scheme 2 , table 1 ). 
<smiles>[Y]c1ccc([N+](=O)[O-])c2ccn(CC)c12</smiles>

4a.f<smiles>[Y]c1ccc([N+](=O)[O-])c2c(C(=O)OCC)cn([R])c12</smiles>

$5 \mathbf{a} \cdot \mathbf{f}$<smiles>[Y]c1ccc([N+](=O)[O-])c2c1N([R])CC2CC(=O)OC</smiles>

$6 a-f$<smiles>[Y]c1ccc2c3c1N([R])CC3CC(=O)N2</smiles>

7a-f

Table 1 : Synthesis of lactam-indolines $7 a-f$

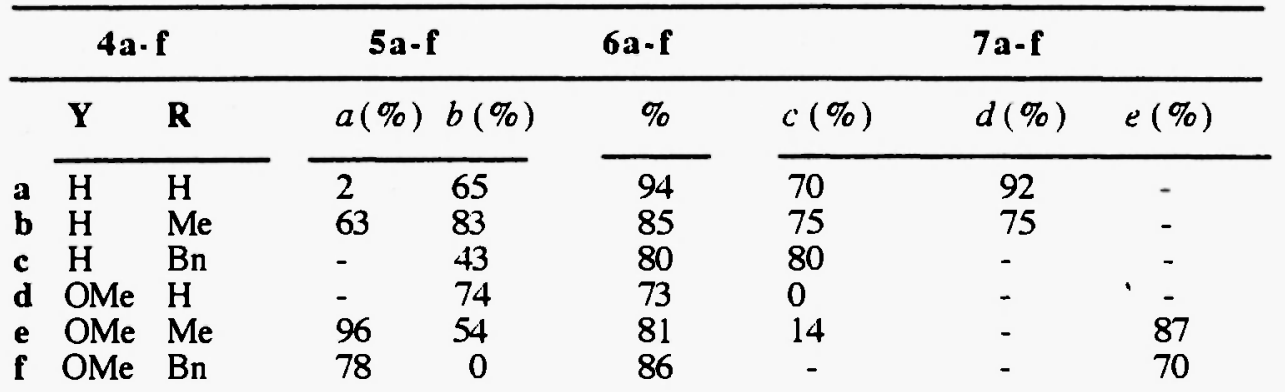

$a:(\mathrm{COCl})_{2}, \mathrm{Et}_{2} \mathrm{O}$, then $\mathrm{MeOH} ; b: \mathrm{P}_{2} \mathrm{O}_{3} \mathrm{Cl}_{4}$, methyl pyrrolidinylglyoxylate, room temperature, $2 \mathrm{~h}$, then $\mathrm{MeOH} ; c: \mathrm{SnCl}_{2}-12 \mathrm{~N} \mathrm{HCl}, 110^{\circ} \mathrm{C}, 3 \mathrm{~h} ; d: \mathrm{Pd}-\mathrm{C}, \mathrm{H}_{2}, 5$ bars , then toluene, $110^{\circ} \mathrm{C}, 12 \mathrm{~h} ; e: \mathrm{PtO}_{2}, \mathrm{H}_{2}$, atm. pressure , then toluene, $110^{\circ} \mathrm{C}, 12 \mathrm{~h}$.

The lactams $7 \mathrm{a}-\mathrm{c}$ were reduced in good yields into amines $9 \mathrm{a}-\mathrm{c}$ by $\mathrm{BH}_{3}-\mathrm{SMe}_{2}$ in THF (Scheme 3).

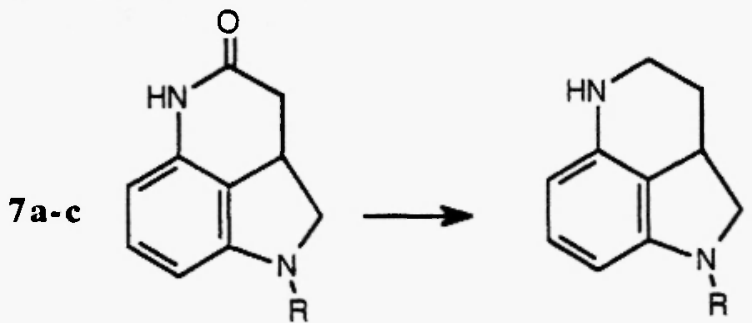

$9 \mathrm{a}: \mathrm{R}=\mathrm{H} 90 \%$

9c $: R=B n 97 \%$

7e-f was methylated by $\mathrm{CH}_{3} \mathrm{I}$ to give $10 \mathrm{e}-\mathrm{f}$ ( $81 \%$ and $90 \%$ respectively) which were reduced with $\mathrm{BH}_{3}-\mathrm{SMe}_{2}$ into $11 \mathrm{e}-\mathrm{f}(88 \%$ and $81 \%) .11 \mathrm{~g}$ was formed from $7 \mathrm{e} \mathrm{(81 \% )} \mathrm{(Scheme} \mathrm{4).}$<smiles>COc1ccc2c3c1N(C)CC3CCN2</smiles>

$11 \mathrm{~g}$<smiles>[R]N1CC2CC(=O)Nc3ccc(OC)c1c32</smiles>

$\mathbf{7 e}: \mathrm{R}=\mathrm{Me}$

7f : $\mathrm{R}=\mathrm{Bn}$<smiles>COc1ccc2c3c1N(P)CC3CCN2C</smiles>

$11 \mathrm{e}: \mathrm{R}=\mathrm{Me}$

$11 f: R=B n$ 
The oxidation of indoline structure is a well-documented reaction. In our hands, the classical rengents (DDQ ${ }^{7}, \mathrm{CuCl}_{2}-\mathrm{O}_{2}{ }^{8}$, cinnamic acid ${ }^{8}$. $\mathrm{PIFA}^{9}$ ) did not give result. Manganese dioxide ${ }^{10}$ gave only oxidation of $7 \mathrm{e}$ at room temperature to lead to the keto-amide $12 \mathrm{a}(42 \%)$. This compound was not reduced into $12 \mathbf{b}$ with $\mathrm{LiAlH}_{4}$. After having successfully used palladium in the presence of ammonium formate ${ }^{3.11}$ to oxidize indolines into indoles this reaction was appliedfor the synthesis of 7e. 9a-b and $11 \mathrm{f}$. Thus, indoles $13 \mathrm{a}-\mathrm{b}$ were obtained with high yields, whereas $11 \mathrm{f}$ led to the debenzylated indole $13 \mathrm{f}$ with moderate yield. $7 \mathrm{e}$ was not oxidized (Table 2).<smiles>[Y]c1ccc2c3c(cn([R2])c13)CCN2P</smiles>

$13 \mathbf{a}, \mathbf{b}, \mathbf{f}$<smiles>COc1ccc2c3c(cn(C)c13)C(=O)C(=O)N2</smiles>

$12 \mathbf{a}$<smiles>COc1ccc2c3c(cn(C)c13)CCN2</smiles>

$12 \mathbf{b}$

Table 2 : Oxidation of indolines into indoles

\begin{tabular}{rlc}
\hline indolin & \multicolumn{1}{c}{ indole } & yield \\
\hline $9 \mathbf{a}$ & $13 \mathbf{a}: \mathrm{R}_{1}=\mathrm{R}_{2}=\mathrm{H}, \mathrm{Y}=\mathrm{H}$ & $90 \% 5$ \\
$9 \mathbf{b}$ & $13 \mathbf{b}: \mathrm{R}_{1}=\mathrm{H}, \mathrm{R}_{2}=\mathrm{Me}, \mathrm{Y}=\mathrm{H}$ & $98 \%$ \\
$1 \mathbf{1 f}$ & $13 \mathbf{f}: \mathrm{R}_{1}=\mathrm{Me}, \mathrm{R}_{2}=\mathrm{H}, \mathrm{Y}=\mathrm{OMe}$ & $67 \%$ \\
$7 \mathbf{e}$ & &
\end{tabular}

Indoline 14a was obtained from $7 \mathrm{f}$ by alkylation with 1-iodo-3-(tetrahydropyran-2yloxy)propane ( $\mathrm{I}-\left(\mathrm{CH}_{2}\right)_{3}$-OTHP) $(98 \%)$, but this compound did not give indole even after long reaction time, only debenzylation into indoline 15 was observed (97\%). The corresponding indole 16a was obtained by oxidation of 15 by the use of Barton reagent (diphenylselenic anhydride) ${ }^{12}$ (41\%) (Scheme 5).

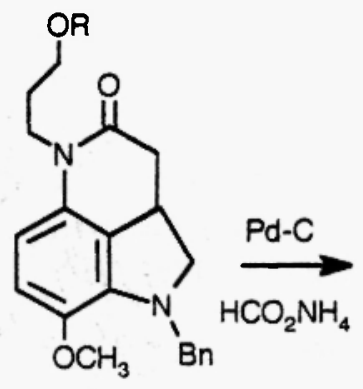

$14 \mathbf{a}: \mathrm{R}=\mathrm{THP}$

$14 b: R=H$<smiles>COc1ccc2c3c1NCC3CC(=O)N2CCC[O-]</smiles>

15<smiles>COc1ccc2c3c(c[nH]c13)CC(=O)N2CCCO</smiles>

$16 a: R=T H P$

16b : $R=H$

Scheme 5

$14 \mathrm{a}$ and $16 \mathrm{a}$ were hydrolyzed in acidic medium into alcohol $14 \mathrm{~b}, 16 \mathrm{~b}(98 \%, 90 \%)$. The treatment of $14 \mathbf{b}$ with $\mathrm{LiAlH}_{4}$ did not give cyclization, only reduction of the carbonyl group was observed to form 18 (32\%) (Scheme 6). Under these conditions $16 \mathrm{~b}$ was not reduced by $\mathrm{LiAlH}_{4}$ and 
Red-Al ${ }^{\circledR}$.The oxazine 19 was obtained by the use of Red-Al ${ }^{(1)}$ from $14 \mathrm{~b}(20 \%)$. Unfortunately we have never obtained indole from 19.

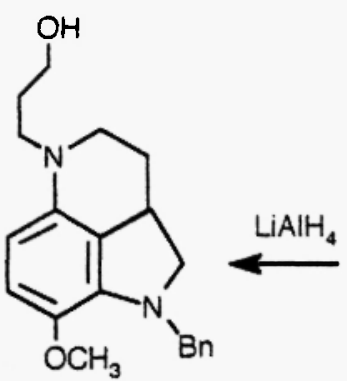

18<smiles>COc1ccc2c3c1N(Cc1ccccc1)CC3CC(=O)N2CCCO</smiles>

$14 b$

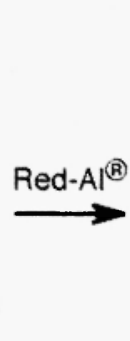<smiles>COc1ccc2c3c1N(Cc1ccccc1)CC3CC1OCCCN21</smiles>

19

Scheme 6

In conclusion, in this work we obtained the precursors of both damirones and haematopodin and now we study the oxidation of these compounds to obtain the natural products.

\section{REFERENCES AND NOTES}

I Current address : Karolinska Institute. Novum, CNT, SE-141 57 Hunninge. Sweden.

1 S. Sakemi, H.H. Sun, C.W. Jefford and G. Bernardenelli, Tetrahedron Lett. 30, 2517 (1989)

2 C. Baumann, M. Brockelmann, B. Fugmann, B. Steffan, , W. Steglich and W.S. Sheldrick. Angew.Chem., Intern. Ed. Engl. 32, 1087 (1993)

3 N. Roue, R. Barret and J. Levy, Synthetic Comm. 25, 681 (1995)

4 J. Bergman and P. Sand, Tetrahedron 46, 6085 (1989)

5 N. Roue, T. Delahaigue and R. Barret, Heterocycles 43, 263 (1996)

6 I.M. Downie, M.J. Earle, H. Heany and K.F. Shuhaibar, Tetrahedron 49, 4015 (1993)

7 E.Walton, F.W. Holly and S.R. Jenkins, J. Org. Chem. 33192 (1968)

8 M. Julia and H. Gaston-Breton, Bull. Soc. Chim. Fr. 1335 (1966)

9 R. Barret, A. Belassaoui, Z.M. Du, T. Mulamba, J.Y. Laronze and J. Lejy, Heterocyclic Comm. 5, 329 (1999)

10 A.B.A. Jansen, J.M. Johnson and J.R. Surtees, J. Chem. Soc. 5573 (1964)

11 B.M. Adger, C. O'Farell, N.J. Lewis and M.B. Mitchell, Synthesis 53 (1987); T. Kiguchi, N. Kininobu, Y. Takahashi, Y. Yoshida, T. Naito and I. Ninomiya, Synthesis 778 (1989)

12 D.H.R. Barton, M.J. Earle, H. Heaney and K.F. Shuhaibar, Tetrahedron 414727 (1985)

13 Physical data of some products : $10 \mathrm{f}: \mathrm{F}: 107-110^{\circ} \mathrm{C}$; $\mathrm{HRMS}:$ calculated for $\mathrm{C}_{19} \mathrm{H}_{20} \mathrm{~N}_{2} \mathrm{O}_{2}$ : 308.1524, found : 308.1522; ' $\mathrm{H}-\mathrm{NMR}\left(\mathrm{CDCl}_{3}, 300 \mathrm{MHz}\right): 2.45(1 \mathrm{H}, \mathrm{t}, \mathrm{J}=15 \mathrm{~Hz}) 2.85(2 \mathrm{H}$, $\mathrm{m}), 3.27(3 \mathrm{H}, \mathrm{s}) 3.59(1 \mathrm{H}, \mathrm{t}, \mathrm{J}=8 \mathrm{~Hz}) 3.81(3 \mathrm{H}, \mathrm{s}) 4.16(1 \mathrm{H}, \mathrm{d}, \mathrm{J}=14 \mathrm{~Hz}) 5.07(1 \mathrm{H}, \mathrm{d}, \mathrm{J}$ $=14 \mathrm{~Hz}) 6.29(\mathrm{lH}, \mathrm{d}, \mathrm{J}=9 \mathrm{~Hz}) 6.71(\mathrm{lH}, \mathrm{d}, \mathrm{J}=9 \mathrm{~Hz}) 7.29-7.31(3 \mathrm{H}, \mathrm{m}) .11 \mathrm{f}:$ HRMS : calculated for $\mathrm{C}_{19} \mathrm{H}_{22} \mathrm{~N}_{2} \mathrm{O}: 294.1731$, found : 294.1732; ${ }^{\mathrm{l}} \mathrm{H}-\mathrm{NMR}\left(\mathrm{CDCl}_{3}, 300 \mathrm{MHz}\right): 1.71$ $(1 \mathrm{H}, \mathrm{m}) 2.06(\mathrm{lH}, \mathrm{m}) 2.64(1 \mathrm{H}, \mathrm{dd}, \mathrm{H}=8$ and $12 \mathrm{~Hz}) 2.84(3 \mathrm{H}, \mathrm{s}) 3.17(2 \mathrm{H}, \mathrm{m}) 3.41(\mathrm{HH}, \mathrm{t}$, $\mathrm{J}=8 \mathrm{~Hz}) 3.74(3 \mathrm{H}, \mathrm{s}) 3.98(1 \mathrm{H}, \mathrm{d}, \mathrm{J}=14 \mathrm{~Hz}) 5.14(\mathrm{lH}, \mathrm{d}, \mathrm{J}=14 \mathrm{~Hz}) 6.03(\mathrm{IH}, \mathrm{d}, \mathrm{J}=9$ Hz) $6.65(1 \mathrm{H}, \mathrm{d}, \mathrm{J}=9 \mathrm{~Hz}) 7.30-7.35(5 \mathrm{H}, \mathrm{m}) .19:{ }^{1} \mathrm{H}-\mathrm{NMR}\left(\mathrm{CDCl}_{3}, 300 \mathrm{MHz}\right): 1.60(2 \mathrm{H}$, m) $1.92(2 \mathrm{H}, \mathrm{m}) 2.66(1 \mathrm{H}, \mathrm{m}) 3.29(\mathrm{lH}, \mathrm{m}) 3.41(2 \mathrm{H}, \mathrm{m}) 3.75(3 \mathrm{H}, \mathrm{s}) 3.92(2 \mathrm{H}, \mathrm{m}) 4.07(2 \mathrm{H}$, m) $6.15(1 \mathrm{H}, \mathrm{d}, \mathrm{J}=8 \mathrm{~Hz}) 6.68(1 \mathrm{H}, \mathrm{d}, \mathrm{J}=8 \mathrm{~Hz}) 7.29-7.31(5 \mathrm{H}, \mathrm{m})$; ${ }^{13} \mathrm{C}-\mathrm{NMR}: 31.1,31.9$, $46.9,54.9,57.9,61.7,68.3,85.4,103.1,114.5,115.0,126.2,128.3,130.1,135.7,139.0$, 139.6 .

Received on February 7, 2000 